\title{
VARIATIONAL ASPECTS OF THE \\ ABEL AND SCHRODER FUNCTIONAL EQUATIONS
}

\author{
M. A. McKiernan
}

(received July 15, 1962)

Given an analytic function $f$, the successive iterates of $f$ are defined by

$$
f^{[0]}(z)=z, f^{[n+1]}(z)=f\left\{f^{[n]}(z)\right\} \text { for every } z .
$$

In particular $f^{[1]}(z)=f\left\{f^{[0]}(z)\right\}=f(z)$. Extensive study has been given [1] to the problem of generalizing the iterates $f^{[n]}$, for integer $n$, to $f^{[t]}$ for arbitrary $t$, where the iterative character of $f^{[t]}$ is to be preserved by the conditions,

$$
f^{[0]}(z)=z \text { and } f^{[s]}\left\{f^{[t]}(z)\right\}=f^{[s+t]}(z) \text { for arbitrary }
$$

$s$ and $t$.

Certain functional equations are closely related to this problem, the most well known [1] being

$$
\begin{aligned}
& g\{f(z)\}=\alpha+g(z) \text {, AbeI's functional equation } \\
& \text { (usually one takes } \alpha=1 \text { ), } \\
& g\{f(z)\}=\alpha g(z), \quad \text { Schröder's functional equation, } \\
& g\{f(z)\}=\{g(z)\}^{\alpha}, \text { Bottcher's functional equation, }
\end{aligned}
$$

where the function $f$ is assumed given and $g$ is the function to be found. Repeated substitutions of $f(z)$ into these equations yields, respectively,

Canad. Math. Bull. vol. 6, no. 2, May 1963. 


$$
\begin{aligned}
& g\left\{f^{[n]}(z)\right\}=n \alpha+g(z), \\
& g\left\{f^{[n]}(z)\right\}=\alpha^{n} g(z), \\
& g\left\{f^{[n]}(z)\right\}=\{g(z)\} \alpha^{n} .
\end{aligned}
$$

In view of the simplicity of the right hand sides of the se equations, it is natural to define generalized iterates of $f$ by means of the equations

$$
\begin{aligned}
& \mathrm{f}^{[\mathrm{t}]}(\mathrm{z})=\mathrm{g}^{[-1]}\{\mathrm{t} \alpha+\mathrm{g}(\mathrm{z})\} \\
& \mathrm{f}^{[\mathrm{t}]}(\mathrm{z})=\mathrm{g}^{[-1]}\left\{\alpha^{\mathrm{t}} \mathrm{g}(\mathrm{z})\right\} \\
& \mathrm{f}^{[\mathrm{t}]}(\mathrm{z})=\mathrm{g}^{[-1]}\left\{[\mathrm{g}(\mathrm{z})]^{\alpha^{\mathrm{t}}}\right\}
\end{aligned}
$$

where $g^{[-1]}$ denotes the inverse function of $g$, (and hence the usual problems associated with inverse functions must be considered).

If $\mathrm{f}^{[\mathrm{t}]}(\mathrm{z})$ is defined for all real $\mathrm{t}$, then each complex number $z=z_{0}$ determines a curve given parametrically in terms of $t$ by $z=f^{[t]}\left(z_{0}\right)$, which will be called the iteration trajectory of $f$ through $z_{0}$. It was noted by Schröder [2] that an analytic solution, $g$, of the Abel or Schröder equation for real $\alpha$, defines a conformal mapping $w=g(z)$ which maps the iteration trajectories of $f$ into straight lines; lines through the origin when $g$ is a solution of Schröder's equation, and horizontal lines when $g$ is a solution of Abel's equation.

Following the vein of Schröder's observation, in this paper we wish to point out a simple but interesting variational relation between the iteration trajectories of an analytic function and the Abel, Schröder, and related functional equations. To begin our discussion, we need the following lemma, which is a particular case of a well known theorem [3] and is given here merely for completeness.

LEMMA 1: The Euler equations for the functional 


$$
\int_{C} G(x, y) \sqrt{x^{2}+\dot{y}^{2}} d t
$$

where $C$ is the curve given by $x=x(t), y=y(t)$, and $G(x, y)>0$, can be written in the form

$$
\left\{\begin{array}{l}
G(x, y) \ddot{x}=\left(\dot{y}^{2}-\dot{x}^{2}\right) G_{x}(x, y)-2 \dot{x} \dot{y} G_{y}(x, y) \\
G(x, y) \ddot{y}=-2 \ddot{x} \dot{y} G_{x}(x, y)-\left(\dot{y}^{2}-\dot{x}^{2}\right) G_{y}(x, y)
\end{array}\right.
$$

when the parameter $t$ is chosen as the arc length along $C$ in the Riemannian geometry determined by (4), (and hereafter designated as the G-length).

Proof: From the definition of $t$ it follows that along $C$

$$
1=\frac{d}{d t}\left\{\int_{t}^{t} G(x, y) \sqrt{x^{2}+\dot{y}^{2}} d t\right\}=G(x, y) \sqrt{x^{2}+\dot{y}^{2}}
$$

The Euler equations for (4) are

$$
\begin{aligned}
& G_{x}(x, y) \sqrt{\dot{x}^{2}+\dot{y}^{2}}-\frac{d}{d t}\left\{\frac{G(x, y) \dot{x}}{\sqrt{\dot{x}^{2}+\dot{y}^{2}}}\right\}=0 \text { and } \\
& G_{y}(x, y) \sqrt{\dot{x}^{2}+\dot{y}^{2}}-\frac{d}{d t}\left\{\frac{G(x, y) \dot{y}}{\mid \sqrt{x^{2}+y^{2}}}\right\}=0,
\end{aligned}
$$

which, making use of (6), become

$$
\begin{aligned}
& G_{x}(x, y)-G(x, y) \frac{d}{d t}\left\{\frac{\dot{x}}{\dot{x}^{2}+\dot{y}^{2}}\right\}=0 \text { and } \\
& G_{y}(x, y)-G(x, y) \frac{d}{d t}\left\{\frac{\dot{y}}{\dot{x}^{2}+\dot{y}^{2}}\right\}=0 .
\end{aligned}
$$

Carrying out the indicated differentiations and solving the system for $\ddot{x}$ and $\ddot{y}$ yields the desired equations (5). 
THEOREM: Let $g$ be an analytic function and set $G(x, y)=\left|g^{\prime}(\xi)\right|$. Then the extremals of the functional

$$
\int_{C} G(x, y) \sqrt{\dot{x}^{2}+\dot{y}^{2}} d t
$$

are explicitly given by

$$
x(t)+i y(t)=g^{[-1]}\{\alpha t+g(z)\} \text { where } \alpha \text { and } z \text { are }
$$

arbitrary constants. Hence every extremal $x(t)+i y(t)$ is the iteration trajectory, through $z$, of an analytic function $f$, where $f$ is defined by

$$
f(z)=g^{[-1]}\{\alpha+g(z)\} .
$$

Moreover, in view of (1), it is clear that $g$ is an Abel function for $f$, and that the index of iteration, $t$, in $f^{[t]}(z)=$ $\mathrm{g}^{[-1]}\{\alpha \mathrm{t}+\mathrm{g}(\mathrm{z})\}$, is the G-length (up to a constant factor) as measured by (7) along the iteration trajectory from $z$ to $f^{[t]}(z)$.

Proof: The modified Euler equations (5) can be written in the form

$$
\ddot{x}+i \ddot{y}=-(\dot{x}+i \dot{y})^{2}\left\{\frac{G_{x}(x, y)}{G(x, y)}-i \frac{G_{y}(x, y)}{G(x, y)}\right\} .
$$

Since $\ln \left|\mathrm{g}^{\prime}(\xi)\right|=\ln \mathrm{G}(\mathrm{x}, \mathrm{y})$ is the real part of the analytic function $\ell n g^{\prime}(\xi)$, it follows that $\frac{\partial}{\partial x}\{\ell n G(x, y)\}$ is the real part, and $-\frac{\partial}{\partial y}\{\ell n G(x, y)\}$ the imaginary part, of the analytic function $\frac{\mathrm{d}}{\mathrm{d} \xi}\left\{\ell \mathrm{n} \mathrm{g}^{\prime}(\xi)\right\}$. Hence $(8)$ becomes

$$
\frac{\ddot{x}+i \ddot{y}}{\dot{x}+i \dot{y}}=-\frac{d}{d \xi}\left\{\ln g^{\prime}(\xi)\right\}(\dot{x}+i \dot{y})=-\frac{d}{d t}\left\{\ln g^{\prime}(x+i y)\right\} \text {. }
$$

Integration yields 


$$
\dot{x}+i \dot{y}=\frac{\alpha}{g^{\prime}(x+i y)} \text { or } g^{\prime}(x+i y)(x+i y)=\alpha,
$$

where $\alpha$ is an arbitrary complex number. A second integration yields

$$
g(x+i y)=\alpha t+\beta
$$

where $\beta$ is an arbitrary complex number which we choose so that $\beta=g(z)$, for arbitrary $z$. It follows that

$$
\begin{aligned}
& x(t)+i y(t)=g^{[-1]}\{\alpha t+g(z)\}=f^{[t]}(z), \\
& \text { where } g\{f(z)\}=\alpha+g(z) .
\end{aligned}
$$

It may readily be verified that equations (5), by multiplying the first by $\dot{x}$ and the second by $\dot{y}$ and adding, imply that along $\mathrm{C}$,

$$
0=\frac{d^{2}}{d t^{2}}\left\{\int_{t}^{t} G(x, y) \sqrt{\dot{x}^{2}+\dot{y}^{2}} d t\right\} .
$$

Hence, in the modified Euler equations (8), the parameter is a constant multiple of the G-length along $C$, and since $f^{[0]}(z)=z$, the theorem follows.

COROLLARY 1: Let $f$ be an analytic function whose corresponding analytic Abel function is $g$, so that $g\left\{f^{[t]}(z)\right\}=$ $\alpha t+g(z)$. Then $f^{[t]}(z)$ is a geodesic in the Riemannian geometry defined by

$$
\int_{C}\left|g^{2}(z)\right| \sqrt{x^{2}+y^{2}} d t \text {, }
$$

and $f(z)$ is merely a uniform translation in this geometry.

Proof: By the theorem $f$ maps $f^{[t]}(z)$ into $f^{[t+1]}(z)$ which is one unit, as measured by $\left(7^{\prime}\right)$, along the geodesic. 
COROLLARY 2: The generalized iterates $f^{[t]}(z)$ of $f(z)$, as defined by the Abel, Schröder, and Bottcher functional equations (1), (2), (3) for arbitrary $\alpha$, are the extremals through $\mathrm{z}$ of the functionals

$$
\begin{aligned}
& \int\left|g^{\prime}(z)\right| \sqrt{\dot{x}^{2}+\dot{y}^{2}} d t, \quad \int\left|\frac{g^{\prime}(z)}{g(z)}\right| \sqrt{\dot{x}^{2}+\dot{y}^{2}} d t, \\
& \int\left|\frac{g^{\prime}(z)}{g(z) \ln g(z)}\right| \sqrt{\dot{x}^{2}+\dot{y}^{2}} d t
\end{aligned}
$$

respectively, with the parameter $t$ as the respective G-length of the iteration trajectory from $z=f^{[0]}(z)$ to $f^{[t]}(z)$.

Proof: Let $g_{A}, g_{S}$, and $g_{B}$ denote the Abel, Schroder, and Bottcher functions for $f^{[t]}(z)$, respectively. Then $g_{B}\left(f^{[t]}(z)\right)=\left[g_{B}(z)\right]^{\alpha}$, and taking the logarithm of both sides yields $\ell n g_{B}\left\{f^{[t]}(z)\right\}=\alpha^{t} \ell n g_{B}(z)$, that is, $g_{S}(z)=\ell n g_{B}(z)$.

Similarly from $g_{S}\left\{f^{[t]}(z)\right\}=\alpha^{t} g_{S}(z)$, it follows on taking Iogarithms, that $\ell \mathrm{ng}_{\mathrm{S}}\left\{\mathrm{f}^{[\mathrm{t}]}(\mathrm{z})\right\}=\mathrm{t} \ell \mathrm{n} \alpha+\ell \mathrm{n} \mathrm{g}_{\mathrm{S}}(\mathrm{z})$. Hence $g_{A}(z)=\frac{\alpha}{\ln \alpha} \ln g_{S}(z)$. Thus

$$
g_{A}(z)=\frac{\alpha}{\ln \alpha} \cdot \ln g_{S}(z)=\frac{\alpha}{\ln \alpha} \cdot \ln \ell n g_{B}(z)
$$

and the corollary follows from Theorem 1 by merely replacing $g_{A}^{\prime}(z)$ by the appropriate expression.

COROLLARY 3: Given the iteration family of functions $f^{[t]}(z)$, let $g_{A}(z)$ and $g_{S}(z)$ be corresponding Abel and Schroder functions, respectively; that is

$$
f^{[t]}(z)=g_{A}^{[-1]}\left\{a t+g_{A}(z)\right\}=g_{S}^{[-1]}\left\{b^{t} \cdot g_{S}(z)\right\}
$$


for real $b>0, b \neq 1$.

Then the curves (iteration trajectories) given parametrically by

$$
z=f^{[t]}\left(z_{0}\right) \text {, for arbitrary fixed } z_{0} \text {, }
$$

are common extremals of the functionals

$$
\int\left|g_{A}^{\prime}(z)\right| \sqrt{\dot{x}^{2}+\dot{y}^{2}} d t, \int\left|g_{S}^{\prime}(z)\right| \sqrt{\dot{x}^{2}+\dot{y}^{2}} d t
$$

where, however, no relation between the $t$ in the Schroder part of (10) and the corresponding G-length is implied.

Proof: As in the proof of theorem 1 the extremals are given respectively by (see (9))

$$
\mathrm{g}_{\mathrm{A}}^{[-1]}\{\alpha \mathrm{t}+\beta\}, \mathrm{g}_{\mathrm{S}}^{[-1]}\{\alpha \mathrm{t}+\beta\}
$$

where $t$ is the G-length as defined by the respective functionals. In the first expression, set $\alpha=a$ and $\beta=g_{A}(z)$. In the second expression replace $t$ by the parameter $\tau$ given by $t=b^{\top}$ and choose $\beta=0 . \alpha=g_{S}(z)$. Since $b>0$ is real, $\tau$ is real. The extremals (11) then become $f^{[\tau]}(z)$ as defined in (10), proving the corollary.

The above analysis may of course be generalized since the Abel, Schröder and Bottcher equations are merely particularly simple examples of the following more general method of finding $f^{[t]}(z)$ for arbitrary $t$. Let $\phi(z)$ be such that $\phi^{[t]}(z)$ is known for arbitrary $t$. If a function $g(z)$ can be found such that

$$
g\{f(z)\}=\phi\{g(z)\} \text {, then } g\left\{f^{[n]}(z)\right\}=\phi^{[n]}\{g(z)\} \text {, }
$$

which suggests the definition

$$
f^{[t]}(z)=g^{[-1]}\left\{\phi^{[t]}(g(z))\right\} .
$$


The Abel, Schroder and Bottcher equations arise when $\phi(z)=\alpha+z, \quad \phi(z)=\alpha \cdot z$, and $\phi(z)=z^{\alpha}$, respectively.

Finally we note that $g\left\{f^{[t]}(z)\right\}=\alpha t+g(z)$, by differentiating both sides with respect to $t$, implies that

$$
g^{\prime}\left\{f^{[t]}(z)\right\} \cdot \frac{\partial}{\partial t} f^{[t]}(z)=\alpha .
$$

Let $t=0$, and let $\left.\frac{\partial}{\partial t} f^{[t]}(z)\right|_{t=0}=L(z)$. Since $f^{[0]}(z)=z$, (12) becomes

$$
g^{\prime}(z)=\frac{\alpha}{L(z)} \text {. }
$$

The function $L(z)$ is used extensively by Jabotinsky [1] in his study of generalized iterates. In particular, differentiating

$$
\mathrm{f}^{[\mathrm{s}]}\left\{\mathrm{f}^{[\mathrm{t}]}(\mathrm{z})\right\}=\mathrm{f}^{[\mathrm{t}]}\left\{\mathrm{f}^{[\mathrm{s}]}(\mathrm{z})\right\}
$$

with respect to $t$ and setting $t=0$ yields

$$
\frac{\partial}{\partial z} f^{[s]}(z) \cdot L(z)=L\left\{f^{[s]}(z)\right\}
$$

whence the one parameter family of functions ( $s$ the parameter) $w=f^{[s]}(z)$ all satisfy the differential equation

$$
L(w)=L(z) \cdot \frac{d w}{d z}
$$

In terms of $L(z)$, the functional (7) becomes

$$
\int_{C}^{\sqrt{\dot{x}^{2}+\dot{y}^{2}}} \frac{}{|L(z)|} d t \text {. }
$$




\section{REFERENCES}

1. See for example, G. Szekeres, Regular Iteration of Real and Complex Functions, Acta Mathematica, 100 (1958), pp. 203-258. Also P. Erdös and E. Jabotinsky, On Analytic Iteration, Journal $D^{\prime}$ Analyse Mathematique, Vol. 8 , part 2, 1960/61, Jerusalem.

2. E. Schröder, Uber iterierte Funktionen, Mzth. Ann., 2(1870) pp. 317-365.

3. The equations are given in tensor form for example in I. S. Sokolnikoff, Tensor Analysis, John Wiley and Sons, 1951 , p. 161.

University of Waterloo 